\title{
El discurso didascálico. Una tipología revisada
}

\section{(4) Mariano Nicolás Zucchi \\ CONICET / Universidad de Buenos Aires / Universidad Nacional de las Artes, Argentina marianonzucchi@gmail.com}

Fecha de recepción: 12/01/2021. Fecha de aceptación: 05/04/2021

\begin{abstract}
Resumen
Las didascalias constituyen, sin dudas, el tipo de enunciado más estudiado por los estudios teatrológicos. Sin embargo, no existe en la actualidad un consenso respecto a las características lingüísticas distintivas de estos discursos ni a su función en el proceso de construcción de sentido. Luego de revisar críticamente las diferentes definiciones que la tradición provee sobre las didascalias, este artículo se propone identificar la naturaleza específica de estas expresiones y sus atributos particulares. Para ello, y frente al reconocimiento de que el discurso didascálico no constituye un conjunto homogéneo de enunciados, se plantea, en primer lugar, la necesidad de establecer una taxonomía que atienda a la diversidad de empleos y de significados que aportan. En ese sentido, se propone aquí distinguir entre las didascalias paratextuales, las estructurales, las generales, las atributivas y las particulares. Mediante la utilización de ejemplos de obras representativas de distintas estéticas teatrales, se explican las propiedades morfo-sintácticas de cada uno de estos subtipos y sus diversas funciones. Finalmente, y en cuanto a la perspectiva de trabajo, se adopta aquí el Enfoque dialógico de la argumentación y la polifonía enunciativa (Caldiz, 2019; García Negroni, 2016a; 2016b; 2018a; 2018b; 2019; García Negroni y Libenson, 2018; 2019).
\end{abstract}

Palabras clave: didascalia, texto dramático, réplica, polifonía, dialogismo

\section{The Discourse of Stage Directions. A Revised Typology}

\begin{abstract}
Stage directions are one of the most studied components of the dramatic text. Nevertheless, there is currently no consensus about their distinctive linguistic characteristics and their role in the meaning construction process. After reviewing the traditional definitions of stage directions, this article aims to identify the specific nature of these expressions and their particular attributes. In order to achieve this objective, we present a taxonomy that addresses the diversity of usages and meanings that these statements provide. In particular, we distinguish between didascalias paratextuales,
\end{abstract}


estructurales, generales, atributivas and particulares. The morpho-syntactic properties of these subtypes and their functions are explained by using examples of representative plays from different theatrical aesthetics. Finally, and regarding the theoretical footing, this paper applies the Enfoque dialógico de la argumentación y la polifonia enunciativa (Caldiz, 2019; García Negroni, 2016a; 2016b; 2018a; 2018b; 2019; García Negroni and Libenson, 2018; 2019) as framework.

Keywords: Stage Directions, Dramatic Text, Dialogue, Polyphony, Dialogism

\section{Introducción}

El discurso didascálico fue tradicionalmente uno de los fenómenos más estudiados en el campo de la teatrología. Su naturaleza particular y el hecho de que estos enunciados aparezcan de forma excluyente en el texto dramático (en adelante, TD) hicieron de las didascalias el objeto de estudio privilegiado de los trabajos que pretenden teorizar sobre la especificidad del género. Sin embargo, y a pesar de la gran cantidad de autores que abordaron el tema, no existe actualmente un consenso sobre los atributos distintivos de estos enunciados ni sobre su función en el proceso de construcción de sentido.

En términos generales, es posible identificar dos grandes tradiciones que se ocuparon de caracterizar a las didascalias: en primer lugar, la que las considera como acotaciones escénicas, esto es, como enunciados especializados en señalar el modo en que un TD debe materializarse en el escenario (cf., entre otros, Andjelkovic, 2008; Bobes Naves, 2004; Conde, 2017; 2018; De Toro, 2008; Fitzpatrick, 1987; Fobbio, 2009; García Barrientos, 2009; 2012; Helbo, 2012; Hermenegildo, 1991; Ingarden 1971; Issacharoff, 1981; Lasso Osorio, 2013; Llagostera, 2015; Pavis, 1988; Petitjean, 2011; Prochazka 1997; Rubio Montaner, 1990; Sanchis Sinisterra, 2002; Thomasseau, 1997; Ubersfeld, 1989; Vaisman, 1979; Zich, 1931); en segundo lugar, aquella que propone que las didascalias se ocupan de dar información sobre el contexto de enunciación en el que se desarrolla el diálogo teatral (cf., entre otros, De Marinis, 2005; Ryngaert, 2004; Schmidhuber de la Mora, 2009; Veltrusky, 1997; Villegas, 1991). Al respecto, es importante mencionar que cada una de estas descripciones es deudora del modo en que cada tradición concibe la naturaleza del género. Así, si se entiende que el TD es un objeto que se orienta hacia su realización espectacular o que depende de la puesta en escena para especificar su sentido, las didascalias tienden a ser caracterizadas como indicaciones escénicas. En cambio, si el TD se percibe como una obra literaria autónoma, estos enunciados se presentan como involucrados en la determinación de las características de la situación dramática en la que se produce el intercambio entre los personajes.

Ahora bien, como se espera mostrar en este trabajo, ambas definiciones son inadecuadas, ya que no dan cuenta de la especificidad de este tipo de discurso. En efecto, la tradición que concibe las didascalias como acotaciones escénicas no puede explicar un número importante de enunciados de este tipo, que no admiten ser interpretados como señalamientos relativos a la manera en la que el TD debe ser llevado a escena. Considérese el siguiente ejemplo, extraído de la didascalia final de la traducción al español de Inventarios de Minyana:

(1) [...] Las cuatro mujeres comen su porción de torta mirando al público... ¿lgor, les habrá dado las gracias? ([1986] 1998:162)

Como puede observarse, el segmento interrogativo en la muestra (1) no funciona como una indicación para la representación, ya que, de hecho, resultaría altamente problemático determinar qué información estaría brindando sobre la construcción efectiva 
de la puesta en escena. Asimismo, el enunciado construye una imagen del lector en tanto alocutario de la pregunta (como aquel a quien se dirige la interrogación), lo que vuelve evidente que el material no está orientado hacia la construcción de un espectáculo, marco en el que por necesidad este fragmento de discurso quedaría excluido.

Además, también resulta imprecisa la caracterización lingüística que esta tradición propone para el discurso didascálico, en particular, el valor apelativo que se le atribuye a estos enunciados (cf., García Barrientos, 2012; Pavis, 1988; Rubio Montaner, 1990; Ubersfeld, 1989; Vaisman, 1979). Para estos autores, en las didascalias hay un predominio de la función conativa (Jakobson, 1975), lo que convierte a estas expresiones en una suerte de exhortación que el dramaturgo le da al equipo de realización del espectáculo. Es más, este carácter instruccional atribuido a las didascalias es el fundamento en el que se apoyan algunos teóricos para justificar sus propiedades lingüísticas específicas. Así, para García Barrientos (2012:55-56), se explicaría el predominio del tiempo presente y la ausencia de forma personales, deícticos, usos interlocutivos y narrativos del lenguaje. Para Vaisman (1979:15), en cambio, es en el valor apelativo de estos discursos en el que se funda la economía, la fragmentariedad y el ascetismo que los caracterizan. De hecho, para el autor, estos elementos vuelven a las didascalias una "semia-sustitutiva", esto es, un lenguaje técnico que repone las características que el diálogo teatral adquiere en la escena.

Sin embargo, si se examinan estos enunciados en términos lingüísticos, la hipótesis del dominio de la función conativa puede ser rápidamente descartada. En efecto, un examen detallado de la materialidad discursiva muestra que las didascalias jamás utilizan verbos en modo imperativo, el cual, como se sabe, es el modo privilegiado del español para construir enunciados con valor apelativo. Más aún, la ausencia de infinitivos en estos enunciados -otra de las formas que suelen adquirir los enunciados instructivos (piénsese, por ejemplo, en una receta de cocina)- también funciona como evidencia que desacredita el hecho de que las didascalias constituyan un acto de orden.

Una crítica similar puede formularse respecto a los trabajos que definen a las didascalias como enunciados que indican el contexto de enunciación en el que se desarrolla el diálogo teatral. Para esta tradición, que entiende que el TD es una obra literaria autónoma, el discurso didascálico no constituye una indicación para la escena, sino que colabora en la construcción de la situación dramática mediante el señalamiento del marco espacio-temporal en el que transcurre la acción y de todos los elementos "no lingüísticos" que acompañan a las réplicas (Villegas, 1991:14).

Si bien este artículo acuerda parcialmente con esta tradición, en particular, con que el TD es un objeto que no depende de su eventual puesta en escena para construir sentido, la definición de los enunciados didascálicos desarrollada en el párrafo anterior es problemática, ya que no explica con nitidez el funcionamiento enunciativo de estas expresiones ni los tipos de significados que aportan. En particular, esta perspectiva tiende a ser bastante general al momento de determinar cuáles son los "elementos no lingüísticos" que repondrían las didascalias. Para Schmidhuber de la Mora, por ejemplo, estos enunciados incorporan información sobre las "las apariencias físicas y emocionales de los personajes" (2009:193). Nótese, al respecto, la oscuridad de la expresión "apariencias emocionales". Otros autores (Ryngaert, 2004; Villegas, 1991), en cambio, señalan que los "aspectos extraverbales" indicados por las didascalias incluirían información quinésica y proxémica, gestual, actitudinal y física (por ejemplo, sobre la apariencia del personaje y su vestuario). Como puede observarse, el número tan abrumador de dimensiones que quedan englobados en esta categoría y su naturaleza diferencial atentan contra todo posible reconocimiento de la especificidad de estos enunciados. Es más, para estos trabajos los fenómenos suprasegmentales también forman parte de los significados "no lingüísticos" señalados 
por las didascalias. Sin embargo, como se sabe, la prosodia tiene un rol determinante en la producción discursiva (cf. Caldiz, 2015; 2019). En ese sentido, su inclusión en el campo de lo extraverbal no hace otra cosa que evidenciar un problema teórico en la especificación de los significados que aportan las didascalias.

Frente al reconocimiento de algunos de estos problemas y de la diversidad de significados que pueden aportar las didascalias, un grupo de investigadores se vieron obligados a proponer la existencia de distintos tipos de enunciados didascálicos (cf. Andjelkovic, 2008; Carrasco Muñoz, 1980; Dompeyre, 1992; García Barrientos, 2012; Hermenegildo, 1991; Issacharoff, 1981; Schmidhuber de la Mora, 2009). Hermenegildo, por ejemplo, distingue entre didascalias y acotaciones. Las primeras serían aquellos elementos intratextuales que funcionarían a modo de paratextos. La nómina inicial de personajes, los señalamientos espacio-temporales en los que se desarrolla la acción y los enunciados que se utilizan para segmentar el texto en actos y escenas constituirían ejemplos de este primer grupo. Llama acotaciones, en cambio, a aquellas expresiones que, a modo de órdenes dadas por el dramaturgo al equipo de realización del espectáculo, indican "ciertos gestos, movimientos, apariciones, desapariciones, acciones de los personajes, exhibición o manipulación de objetos, etc." (1991:12). Como puede anticiparse, desde la perspectiva adoptada en este trabajo, esta clasificación es imprecisa. Recuérdese, al respecto, la crítica realizada al supuesto valor conativo del discurso didascálico.

Dompeyre, por su parte, propone una tipología con un número extenso de subcategorías, que pretende, justamente, contemplar la diversidad de significados que aportan este tipo de discursos. Así, distingue entre didascalias introductorias (título y anotación del género), introductorias especificas (que señalan el marco espacio-temporal de toda la pieza), intermediarias (que dividen la obra en secuencias e indican el contexto en el que se produce la acción en cada una de ellas), de comienzo de escena (que identifican a los protagonistas de cada macrosecuencia), internas a la escena (en las que se encuentran los enunciados que señalan la atribución del turno de habla, el destinatario de la réplica, el decorado, el vestuario y los objetos presentes, los movimientos y gestos de los personajes, los rasgos prosódicos del diálogo teatral (entonación, volumen, articulación de la voz) y el estado psicológico y actitudinal de los protagonistas) y entre los textos (en las que el dramaturgo comenta la situación de enunciación teatral, por ejemplo, mediante glosas que ajustan el sentido de una expresión o que adaptan la acción al marco espectacular). Si bien esta clasificación tiene la ventaja de proveer un muestrario detallado de las distintas funciones que posee el discurso didascálico, en tanto tipología, posee algunos problemas metodológicos. En principio, engloba en una única clase elementos disímiles (por ejemplo, la convivencia de señalamientos contextuales y elementos de la prosodia en las didascalias internas a la escena). No obstante, la dificultad principal radica en que Dompeyre no propone ningún tipo de criterio formal que permita asignar con precisión un enunciado didascálico a alguna de las categorías. Así, por ejemplo, la didascalia presente en (2), ejemplo extraído de la traducción española de El cuidador, de Pinter, podría ser considerada tanto una didascalia interna a la escena como una didascalia entre los textos:

(2) Davies: No, no está mal este par. (Camina con esfuerzo por el cuarto) Y son fuertes. Tampoco son feos. El cuero es resistente, ¿eh? Muy resistente. ([1959] 2005:26)

En efecto, el enunciado entre paréntesis en la muestra está realizando simultáneamente dos de las funciones asignadas por el autor a dos tipos distintos de didascalias. Si se adopta su perspectiva, el ejemplo en cuestión puede leerse tanto como una 
indicación proxémica o como un señalamiento en torno a la acción física que deben realizar los actores en la escena.

Finalmente, Andjelkovic propone una serie de distinciones que buscan explicar los diversos empleos que hace un TD de las didascalias. En primer término, postula la existencia de didascalias marco, categoría en la que quedaría incluido el título de la pieza, la designación genérica, dedicatorias, prefacio, lista de personajes y la determinación general del espacio y del tiempo del universo de ficción. En segundo término, la autora señala la importancia de distinguir entre didascalias de ilusión escénica y didascalias de ilusión dramática. Esta oposición pretende diferenciar los usos literarios del discurso didascálico de aquellos casos en los que estos enunciados parecerían estar funcionando como acotaciones escénicas. En tercer término, propone una diferencia entre didascalias independientes (ubicadas al comienzo de cada acto o escena) y didascalias dependientes, enunciados que acompañan los discursos de los protagonistas y se especializan en "la recepción fónica de las réplicas de los personajes y la visualización de acciones" (2008:123). Con todo, la propuesta de Andjelkovic, si bien aporta algunas ideas productivas para el análisis de un TD, no posee un criterio de clasificación uniforme. En efecto, la diferencia entre las categorías se apoya en argumentos de distinta índole: sintáctico (para distinguir entre didascalias independientes y dependientes), funcional (al proponer la categoría de didascalia marco) y temático (para diferenciar didascalias de ilusión escénica de las de ilusión dramática). Es más, el hecho de que la taxonomía propuesta no posea una fundamentación homogénea hace que un mismo enunciado didascálico, al igual que lo analizado más arriba, pueda pertenecer a más de una categoría.

Nótese, al respecto, cómo en las tres clasificaciones presentadas persiste en algún grado la concepción de las didascalias como acotaciones escénicas. Si bien los autores hacen un esfuerzo por explicar la diversidad de significados que aportan estos enunciados, su orientación hacia la futura escena, discutida más arriba, siempre está presente. Esto, desde la perspectiva que aquí se defiende, es la causa principal de que las taxonomías no terminen de determinar la especificidad de este tipo de discursos. Asimismo, ninguno de los casos presentados se dedica a justificar la división en clases con un criterio formal uniforme. En consecuencia, la casi nula descripción lingüística que se da de los enunciados didascálicos vuelve muy difícil alcanzar una clasificación ajustada que dé cuenta de sus funcionamientos enunciativos.

Frente a este panorama, este artículo busca explicar la naturaleza particular del discurso didascálico y sus diversas funciones en el proceso de construcción de sentido de todo TD. Para ello, en una primera instancia, se debe hacer notar que las didascalias no constituyen un conjunto homogéneo de enunciados. Por el contrario, existen una serie de diferencias formales y funcionales que vuelven necesario el establecimiento de distintas subcategorías. En ese sentido, este trabajo pretende proponer una taxonomía original del discurso didascálico, una que atienda a las particularidades lingüísticas de este tipo de expresiones. Específicamente, se intentará realizar una distinción entre las didascalias paratextuales (externas a la narración), las didascalias estructurales (especializadas en señalar el modo en que se organiza la acción en el TD), las didascalias generales (enunciados encargados de construir una representación del universo de ficción), las didascalias atributivas (aquellas que explicitan la identidad del responsable de la enunciación de cada turno) y las didascalias particulares (expresiones que acompañan a las réplicas y colaboran en la definición de la escenografía (Maingueneau, 2002) de los diálogos). Al respecto, en Zucchi (2019) se formuló un primer esbozo de esta clasificación. Aquí, en cambio, se presenta una versión revisada y ampliada, ajustada a los últimos avances que tuvo la investigación de doctorado de la que se desprende este trabajo ("El ethos autoral en la Heptalogía de Hieronymus Bosch de Rafael Spregelburd. Subjetividad autoral en 
el texto dramático desde una perspectiva polifónica y dialógica de la enunciación". Doctorado en Artes, Universidad Nacional de las Artes). Con todo, es importante aclarar que, como las didascalias rara vez fueron percibidas como un conjunto heterogéneo de enunciados, no existe una normativa que regule su funcionamiento textual. En ese sentido, y a los efectos de la realización de esta taxonomía, se toma como referencia la manera en que opera el discurso didascálico en la mayoría de los textos consultados.

Ahora bien, dejando de lado las particularidades formales de cada categoría, que serán descriptas más abajo, es importante decir en principio que los cinco subtipos comparten la característica de ser enunciados metadiscursivos, esto es, que son discursos que funcionan como comentarios relativos a la organización del texto, a sus procedimientos constructivos, a las particularidades del mundo de ficción y a las réplicas atribuidas a los personajes. Es más, en la medida en que las didascalias se especializan en modalizar la situación dramática, estos enunciados cumplen un rol fundamental en la definición del sentido global del texto, de la perspectiva enunciativa y de la voz a cargo del discurso (i.e., el ethos autoral).

En cuanto al criterio de clasificación utilizado, se observará que cada una de las categorías aquí presentadas, además de ser enunciados con características morfosintácticas específicas, se diferencia una de otra por la cualidad y extensión del objeto que comentan: en el caso de las didascalias paratextuales es la obra entendida como totalidad; en las estructurales, la organización del entramado textual; en las generales, el conjunto de réplicas sobre las que operan (ya sea a nivel acto, escena/cuadro o secuencia); en las atributivas, el turno de habla; y en las particulares, dependiendo de su posición, es la intervención completa o un fragmento de ella.

Finalmente, en lo relativo a la perspectiva de trabajo adoptada, esta clasificación se propone utilizando el andamiaje teórico que provee el Enfoque dialógico de la argumentación y la polifonía (Caldiz, 2019; García Negroni, 2016a; 2016b; 2018a; 2018b; 2019; García Negroni y Libenson, 2018; 2019), teoría no veritativista ni referencialista de la significación y no unicista del sujeto.

En lo que sigue, luego de presentar mínimamente el marco teórico empleado (cf. \2.), se describirán cada una de las categorías y se analizarán algunos ejemplos que muestren su funcionamiento enunciativo (cf. S3.). Finalmente, se presentan algunas conclusiones (cf. S4.).

\section{Marco}

El Enfoque dialógico de la argumentación y la polifonía (Caldiz, 2019; García Negroni, 2016a; 2016b; 2018a; 2018b; 2019; García Negroni y Libenson, 2018; 2019, en adelante, EDAP) es una perspectiva teórica de orientación no referencialista ni veritativista de la significación y no unicista del sujeto que se presenta como una reformulación de los postulados centrales de la semántica argumentativa francesa (Anscombre y Ducrot, 1994; Carel y Ducrot, 1995; Ducrot, 1984) leídos a la luz de la propuesta bajtiniana (Bajtín, [1979] 2002).

En particular, el EDAP, en sintonía con las ideas de Anscombre y Ducrot, concibe el significado lingüístico en términos argumentativos. Dicho de otro modo, entiende que el sentido de una determinada palabra o expresión no se define por su valor referencial (por las cosas, los hechos o las propiedades que denotan) ni cognitivo (por los pensamientos o creencias que sugieren), sino por aquellos encadenamientos argumentativos que esa entidad evoca (García Negroni, 2016b:282-283). En cuanto 
a estos, Carel y Ducrot (2005) los definen como la combinación de dos y solo dos segmentos unidos por un conector. Este último puede ser de dos tipos: normativo (e.g., "por lo tanto" (PLT)) o transgresivo (e.g., "sin embargo" (SE)). Por ejemplo, y en el marco de la Teoría de los bloques semánticos, el sentido de la expresión "Hace calor. No salgamos a pasear" se define por la evocación del encadenamiento "calor PLT neg. salir", en el que se construye una representación del calor como agobiante, argumento en el que se funda la idea de evitar el paseo.

Además, el EDAP retoma de la Teoría de la polifonía (Ducrot, 1984) la idea de que el sujeto empírico no es el origen ni el responsable del sentido y que sus intenciones no quedan reflejadas en el enunciado. Por el contrario, para esta perspectiva la subjetividad no es otra cosa que una representación que queda plasmada en el discurso del responsable de la enunciación (denominado Locutor (L)). Esta persona del discurso emerge siempre posicionado de formas diversas frente a los puntos de vista (PdV) que son convocados por la expresión. Al respecto, el EDAP redefine la noción de punto de vista ducrotiana y la presenta como un encadenamiento argumentativo que señala el modo en el que queda representada una situación o una entidad en el enunciado. Por ejemplo, en la expresión "El vestido es barato. Compralo" puede identificarse la plasmación de un PdV del tipo "barato PLT comprar" con el que el Locutor queda homologado, lo que muestra que el enunciado se orienta hacia discursos vinculados con la compra del producto.

También el EDAP mantiene de la polifonía ducrotiana la distinción entre L (locutor en tanto responsable de la enunciación) y $\lambda$ (locutor en tanto ser del mundo). De este modo, este marco teórico busca explicar aquellos casos en los que una expresión construye dos imágenes de su productor: una como entidad a cargo de la enunciación; otra como individuo que forma parte de la representación de la realidad que evoca la expresión (y a quien remiten las marcas de primera persona).

No obstante, el EDAP recupera la noción de dialogismo bajtiniana y la ubica en el centro de la descripción semántica. En efecto, y a diferencia de las teorías ducrotianas, esta perspectiva busca explicar cómo los enunciados se insertan en la red interdiscursiva y cómo dicha inserción cumple un rol fundamental en la construcción del sentido y de la (inter)subjetividad (García Negroni, 2019:526). Para ello, el EDAP propone que las instrucciones semánticas asociadas a las palabras y enunciados instan siempre a recuperar enunciaciones otras frente a las cuales la expresión establece un posicionamiento responsivo. Esta reacción de respuesta, por su parte, no solo determina la emergencia de la subjetividad, sino que, además, explica las características que asume la enunciación.

En particular, el EDAP se especializa en el estudio de las indicaciones causales, estas son, los señalamientos que provee el enunciado sobre los motivos de su irrupción. Al respecto, este enfoque sugiere que estas instrucciones obligan a recuperar un marco de discurso que se muestra como la causa sobre lo que se funda la enunciación. Definidos por García Negroni (2016a:9) como los encadenamientos argumentativos "que se presentan como el lugar a partir del cual surge o se desencadena la enunciación actual", los marcos de discurso constituyen una representación argumentativa de la causa del habla $\mathrm{y}$, en ese sentido, explican las propiedades que esta reviste (distanciada, refutativa, asertiva, precavida, entre otras).

En relación con este punto, Maingueneau (2002:61), desde una perspectiva teórica similar, también se pregunta por los modos en que un enunciado legitima y explica su irrupción. Para dar cuenta de este aspecto de sentido, el autor propone el concepto de escenografía, que define como "la escena de habla que el discurso presupone para poder ser enunciado y que este debe validar a través de su enunciación misma". En 
otras palabras, la escenografía constituye una suerte de telón de fondo, construido en la propia enunciación, que incluye una representación de la situación de enunciación, una imagen del vínculo entre los participantes y toda una serie de perspectivas enunciativas que aparecen para darle legitimidad a aquello que se "dice". Al respecto, desde el EDAP, puede pensarse esta dimensión en términos de un conjunto de PdV mostrados que aparecen para enmarcar la expresión, indicar las características que esta reviste y validar las perspectivas enunciativas que se articulan en el plano de lo dicho. Como se observará más adelante, las didascalias particulares constituyen un subtipo de enunciado didascálico especializado en reponer este aspecto del sentido en las réplicas de los personajes.

\section{Tipos de didascalias}

Como quedó dicho, a continuación, se presentarán las cinco subcategorías que conforman la clasificación del discurso didascálico que aquí se propone. En particular, se describirán las características morfo-sintácticas de cada una de ellas, esenciales para su identificación. Luego, se explicará su funcionamiento semántico-enunciativo y se plantearán una serie de ejemplos que buscan ilustrar el rol que estos enunciados tienen en el proceso de construcción de sentido de todo TD.

No obstante, antes de comenzar con la exposición, se considera importante aclarar que los ejemplos que serán utilizados para caracterizar cada una de las categorías buscan ser lo más heterogéneos posibles (esto es, pertenecer a piezas de distintas tradiciones teatrales y de diferentes períodos). Así, se pretende mostrar que la taxonomía presentada se ajusta a las diversas manifestaciones que el género tuvo a lo largo de la historia. Asimismo, vale decir que, por una cuestión de claridad expositiva, se decidió trabajar con ejemplos traducidos al español. En ese sentido, y como toda traducción constituye un fenómeno de reescritura (López, 2013), el análisis que se propone de la muestra no debería hacerse extensivo al original. Además, es importante decir que, en todos los casos, las negritas son nuestras y resaltan algún aspecto de los enunciados que será retomado en la explicación.

\section{Didascalias paratextuales}

La taxonomía aquí descripta denomina didascalias paratextuales (en adelante, DPtx) a aquellos enunciados que se ubican en los márgenes de un TD y funcionan, esencialmente, a modo de paratextos (Genette, 1989). En otras palabras, estas expresiones se especializan en generan un marco de lectura mediante la anticipación de ciertos elementos que se muestran necesarios para la comprensión de la obra. En ese sentido, el hecho de que estas piezas de discurso se orienten a señalar el lugar desde el cual el texto elige ser leído las convierte en un espacio fundamental al momento de determinar la representación que el TD construye de su lector modelo (Eco, 1981, en adelante, LM). En particular, forman parte de las didascalias paratexuales la lista de personajes que intervienen en la acción (y la descripción que muchas veces acompaña al nombre propio), alguna indicación general respecto a las coordenadas espacio-temporales de la obra y los datos que muchos TD brindan sobre su estreno o sobre sus circunstancias de escritura y publicación. En cuanto a estos últimos, en general, funcionan como un mecanismo mediante el cual el material busca acrecentar su grado de legitimidad.

Antes de analizar algunos ejemplos, es importante realizar la siguiente aclaración: no deben confundirse las DPtx con los paratextos propiamente dichos (e.g., título, subtítulo, prólogo, epílogo, portada, entre otros). En efecto, estos últimos, al menos 
en el sentido que los define Genette, no forman parte de la obra en sí y, además, en algunos casos poseen como responsable enunciativo a una persona diferente al autor (en tanto ethos autoral).

A continuación, se presentan dos casos de DPtx con el objetivo de puntualizar algunas de sus características específicas:

(3) Personajes

Doctor Stockmann, médico de un balneario

Señora Stockmann, su esposa.

Petra, su hija

Ejllf, hermano de Petra [...]

Gente del pueblo, hombres de todas las clases sociales, mujeres, escolares.

La acción transcurre en un pueblo de la costa del sur de Noruega. Época actual.

(Ibsen, [1882] 2008:7)

(4) PERSONAJES

Wang, aguatero

Los tres dioses

Shen-Te/Shui-Ta

Yang Sun, aviador sin trabajo [...]

El policía

El vendedor de alfombras y su mujer

Prostituta joven

Prostituta vieja

El barbero Shu-Fu

El bonzo

El desocupado

El mozo de café

Los transeúntes del prólogo

La acción transcurre en la ciudad medio europeizada Se-Chuan. (Brecht, [1943]

1967:4)

En (3), muestra extraída de la traducción al español de Un enemigo del pueblo de Ibsen, aparecen dos DPtx: una en la que se enumera la lista de los personajes que intervienen en la situación dramática y se provee una mínima descripción sobre ellos; otra en la que se señala el marco general en el que transcurre la acción. En cuanto a la primera, es interesante notar el funcionamiento de las glosas que acompañan a los nombres propios. Todas ellas indican que entre los protagonistas se establecen vínculos de parentesco. Así, y en tanto enunciados que cumplen la función de anticipar algunos elementos de la trama y enmarcarlos, estas didascalias advierten que el núcleo del drama se desarrollará en torno al universo familiar, rasgo típico del drama burgués, subgénero al que adscribe el material. Así, la enumeración de los protagonistas ya estaría funcionando como un elemento que comienza a configurar la adscripción de la obra al subgénero. En cuanto a la segunda, resulta importante, en función de los objetivos de este artículo, llamar la atención sobre la presencia del adjetivo con valor deíctico actual. Al respecto, recuérdese que buena parte de la bibliografía especializada señala que las didascalias no poseen entre sus rasgos lingüísticos prototípicos shifters y marcas de subjetividad. Así, la presencia de este deíctico en una obra canónica del siglo XIX muestra, en refuerzo a lo que se argumentó más arriba, cómo los estudios teatrológicos reseñados tienden a partir de una descripción imprecisa de las características de estos discursos al momento de definirlos.

Por su parte, (4), fragmento de la versión española de El alma buena de Se-Chuan de Brecht, aporta una serie de enunciados didascálicos muy productivos a los efectos de 
analizar cómo la pieza anticipa su reproducción del modelo del teatroépico. En primer lugar, la asignación de una doble identidad para la protagonista ("Shen-Te/Shui-Ta") predice que el TD no apostará a un modo realista de construcción de personajes, en el que estos se crean respetando una serie de procedimientos orientados a garantizar que se los perciba como verosímiles, esto es, como personas pertenecientes al mismo universo del que forma parte LM. En esta obra, en cambio, el personaje principal modifica su identidad en varias oportunidades, desplazándose, incluso, de un género a otro. La DPtx, de algún modo, anticipa el movimiento.

En segundo lugar, y en relación con lo anterior, la denominación del resto de los personajes por su rol social (y no por su nombre propio) también muestra la adscripción de la obra a la lógica del teatro brechtiano. En efecto, y al igual que sucede en buena parte del teatro de vanguardia, esta tradición teatral se vale de la lógica del personaje función (cf. Abirached, 1994) para crear a los individuos que forman parte de la situación dramática. En este esquema, los protagonistas son presentados como figuras planas, sin profundidad psicológica y, así, se busca evitar que el lector desarrolle un vínculo de identificación con ellos. El hecho de que estos estén nombrados de este modo por las DPtx no solo anticipa el proceso, sino que ya ubica al LM en un posicionamiento de distancia respecto al universo de ficción.

En tercer lugar, el sintagma "medio europeizada" del enunciado final de la muestra tiene un rol fundamental en la construcción del marco de lectura del TD. Al respecto, es importante decir que la situación dramática transcurre en el pueblo ficcional de Se-Chuan, lugar ubicado en algún país oriental no especificado. Sin embargo, la atribución a este espacio de características europeas funciona como una clave de lectura que permite reconocer que, si bien la obra parecería estar remitiendo a un universo ajeno a su LM (i.e., un individuo europeo), en el fondo, la fábula presentada se vincula directamente con él. Así, se subraya la presencia del procedimiento de la parábola, otro de los rasgos dramatúrgicos distintos del teatro brechtiano.

Como puede observarse, en ambos casos las didascalias paratextuales cumplen un rol fundamental en la construcción del sentido: a partir de la anticipación de algunos elementos de la trama, señalan la adscripción genérica de la obra y crean un marco en el que el LM queda ubicado con una posición específica frente al universo de ficción.

\section{Didascalias estructurales}

Se denomina aquí didascalias estructurales (DE) a aquellos enunciados que encabezan cada acto, cuadro, escena o episodio y que indican de qué tipo de secuencia se trata. En cuanto a su función, estas didascalias sirven no solo para segmentar la pieza en partes, sino también para indicar la jerarquía que se establece entre cada una de ellas. En lo relativo a sus características lingüísticas distintivas, en general están formadas por un sustantivo que señala la naturaleza del fragmento seguido de una indicación numérica (e.g., "Escena 3"). No obstante, en algunos casos, el sintagma nominal puede estar ausente y el dígito ser la única evidencia que señala el límite entre las dos secuencias. Esto es lo que sucede, por ejemplo, en Chau Misterix, de Kartun ([1980] 2006). Otras piezas, como Marat-Sade, de Weiss ([1964] 2010), optan por DE más elaboradas, en las que no se aclara el tipo de fragmento del que se trata, sino que se brinda una especificación sobre su contenido (i.e., a modo de título: "Segunda visita de La Corday" (73)). Así, si bien la función principal sigue siendo la de estructurar la acción, en estos TD, las DE adquieren un valor paratextual, ya que funcionan como elementos que anticipan información. Finalmente, es importante decir que la presencia de este tipo de didascalias no es obligatoria. En efecto, en 
muchos textos el diálogo teatral presenta continuidad de principio a fin (considérese Final de partida, de Beckett ([1957] 2006), o Señorita Julia, de Strindberg ([1889] 2011)).

En cuanto a la productividad de las $\mathrm{DE}$ en la construcción de sentido, la información que aportan es esencial al momento de determinar el tipo de estructura dramática al que apuesta la pieza. Esto, por su parte, puede colaborar en la identificación de otros procedimientos y de la orientación general del texto. Así, por ejemplo, si las DE señalan que el TD se divide en cuadros, definidos por la introducción de nuevas coordenadas espacio-temporales, podrá inferirse que la obra no respeta la unidad de tiempo aristotélica. En última instancia, este dato podría mostrar que la pieza en su totalidad no reproduce el esquema clásico de construcción dramatúrgica (el llamado teatro dramático). Si, en cambio, las $\mathrm{DE}$ presentan el sustantivo episodio, esto indicaría que el TD se ajusta a la lógica de construcción del teatro épico, en la que la estructura dramática está compuesta por unidades autónomas y relativamente independientes entre sí.

\section{Didascalias generales}

La clasificación que aquí se propone establece que existe un tercer tipo de enunciado didascálico: las didascalias generales (DG). Estas expresiones son aquellas que se ubican al inicio y al final de cada acto, cuadro, escena o episodio o entre las réplicas de los personajes. En cuanto a sus rasgos lingüísticos distintivos, las DG tienen autonomía sintáctica (constituyen oraciones completas) y suelen aparecer en itálicas. Además, en términos generales, están escritas en tercera persona del singular de la voz activa del tiempo presente del modo indicativo y tienden a evitar el uso de subjetivemas y expresiones de fuerte carga valorativa.

En cuanto a su función, las DG se especializan en construir una representación del universo de ficción, indicando, básicamente, las características del contexto en el que se produce el diálogo ficcional (se desarrolle este en el marco de un escenario o en un espacio no teatral). Asimismo, estos enunciados aportan información sobre la situación de enunciación del intercambio (Maingueneau, 1999; 2002), específicamente, colaborando en la construcción de la escena englobante (el tipo de discurso al que adscribe el intercambio entre los personajes) y la escena genérica (el género involucrado), aspectos del sentido que, como se detallará en (\$3.5.), en muchos casos las réplicas no pueden precisar por sí mismas. Considérese, por ejemplo, la siguiente DG: "Una oficina. Un jefe y su empleado". En la muestra, se estaría señalando no solo el lugar ficticio en el que se produce el diálogo, sino también, por nombrar solo una posibilidad, que la enunciación del personaje subordinado debe leerse como cortés producto de la asimetría en el poder relativo que se establece entre los participantes.

A continuación, se presenta el análisis de una DG efectiva a los efectos de explicar su rol en la construcción de sentido:

(5) La acción transcurre fundamentalmente en una casona antigua de barrio [...] La obra se inicia un día de semana, aproximadamente a las ocho de la noche. Están en la cocina: María, que pela arvejas frente a una enorme olla; Anyula, que ceba mate, y la Nona. Esta última está sentada en una silla y come pochoclo en forma continuada. Finalmente, Chicho, en su pieza, está tirado en la cama leyendo el diario el día. Anyula le tiende un mate a María.

María: No quiero más.

Anyula: Le voy a llevar a Chicho [...]

María: Dígale que es el último. (Cossa, [1976] 2000:69-70) 
Como puede observarse, en (5), DG inicial con la que comienza La nona de Cossa, se evocan las características principales del marco espacio-temporal en el que se desarrolla la situación dramática ("casa antigua de barrio", "ocho de la noche", "cocina"). Además, y este es el punto que interesa particularmente destacar, los datos aportados por la didascalia ("pela arvejas", "ceba mate") son esenciales para reconocer, al menos en una primera instancia, que el diálogo entre los personajes se produce en un registro extremadamente coloquial. Nótese, al respecto, que las particularidades de la situación de enunciación, fundamentales en el proceso de asignación de sentido, serían imposibles de identificar sin la presencia de la DG.

Finalmente, las DG, en la medida en que se especializan en plasmar una imagen particular del universo de ficción, son el espacio del TD en que se vuelve más evidente la perspectiva de la narración (i.e., el modo y el lugar desde el cual se introduce la acción dramática). En ese sentido, el análisis de marcas de subjetividad que aparecen en estos enunciados resulta productivo al momento de determinar el posicionamiento que asume el TD frente a la situación que presenta y la representación que queda plasmada del ethos autoral en el texto.

\section{Didascalias atributivas}

Se denomina aquí didascalias atributivas (DAt) a aquellos nombres propios o sintagmas nominales que, seguidos del signo "dos puntos", introducen cada una de las réplicas de los personajes. En términos tipográficos, estos enunciados pueden estar escritos tanto en minúsculas como en mayúsculas, dependiendo del criterio editorial. Como se anticipó, aparecen para determinar la identidad del Locutor de cada uno de los diálogos.

Por cierto, salvo contadas excepciones (e.g., 4.48 psicosis de Kane ([1999] 2009)), su presencia es obligatoria en todo TD. En efecto, sin las DAt sería imposible reconocer quién es el individuo responsable de cada intervención y, en consecuencia, sería extremadamente difícil poder comprender la interacción.

\section{Didascalias particulares}

La última categoría que forma parte de la clasificación aquí presentada es la de didascalia particular (DP), enunciados que suelen estar escritos entre paréntesis y acompañan a las réplicas imputadas a los personajes. Estas didascalias, en general, no tienen autonomía sintáctica. En efecto, se caracterizan por presentar fragmentos oracionales en los que tiende a emplearse el participio ("angustiado"), el gerundio ("gritando"), adjetivos ("feliz"), sintagmas preposicionales ("con cariño", "a Juan"), adverbios ("amablemente"), verbos intransitivos ("se sienta") y, en algunos casos, sustantivos o sintagmas nominales que remiten a la prosodia ("pausa") o a la proxemia ("aparte").

Para poder comprender su funcionamiento enunciativo, sin embargo, es necesario explicar una de las características principales de las réplicas, que, llamativamente, es en general pasada por alto por la bibliografía especializada: el hecho de que los diálogos emulen un intercambio lingüístico eminentemente oral, pero, para ello, se valgan del código de la escritura. Como se sabe, en la comunicación lingüística oral intervienen una serie de elementos (e.g., curvas de entonación, gestos, ritmos, volúmenes de voz) que no pueden ser repuestos mediante una transcripción escrita de aquello que dice el personaje. Para resolver esta limitación, el TD se vale del discurso didascálico, en particular, de las DP, enunciados que cumplen un rol fundamental en la construcción de este "simulacro de oralidad". En términos lingüísticos, este tipo 
de didascalias establecen un claro vínculo dialógico con la porción de discurso que modifican. En efecto, se especializan en introducir un PdV que, además de describir algún aspecto de la situación dramática, funciona como un marco a la luz del cual debe leerse la réplica. En otras palabras, las DP especifican el sentido del discurso del personaje mediante la incorporación de una perspectiva enunciativa que en la comunicación oral se evocaría a nivel suprasegmental. Específicamente, estos PdV determinan la escenografía de las réplicas, esto es, la representación de escena de habla instituida por el propio discurso y que funciona para validar el decir (García Negroni, 2018a:229). Como se mostrará más abajo, reponer esta dimensión es esencial para identificar el sentido de los diálogos.

Ahora bien, una taxonomía completa del discurso didascálico debería atender al hecho de que las DP no constituyen tampoco un conjunto homogéneo de enunciados en lo relativo a la posición que adoptan en torno a las réplicas. En efecto, se considera aquí importante distinguir entre las didascalias particulares externas (DPE, ubicadas entre el nombre del personaje y el signo "dos puntos"), las didascalias particulares internas (DPI, que se encuentran dentro de la réplica y la segmentan en partes) y las didascalias particulares finales (DPF, localizadas luego de que el turno de habla haya concluido).

En cuanto a su función, las tres categorías incorporan perspectivas enunciativas que colaboran en la definición de la escenografía de la réplica sobre la que operan (tal como se explicó más arriba). Su diferencia radica, justamente, en el alcance de su intervención. En el caso de las DPE, el PdV presentado afecta a toda la enunciación del personaje; en las DPI, al fragmento de discurso que se encuentra a derecha de la didascalia; en las DPF, al turno siguiente. Nótese, en relación a la hipótesis aquí presentada, que las DP se encuentran siempre antes de la porción de discurso sobre la que estarían operando. Esta posición es fundamental para la construcción de sentido, ya que, si efectivamente colaboran en la evocación de la escenografía de la réplica, es coherente que aparezcan a su izquierda para que el lector tenga información sobre la escena de habla a la luz de la cual debe interpretar el decir del personaje.

Finalmente, y en la medida en que las DPI y las DPF se ubican en el interior de la réplica, también establecen un vínculo dialógico con los fragmentos de discurso que las anteceden. Así, estas didascalias cumplen la función adicional de operar sobre la orientación del enunciado previo, reforzando o modificando la dirección de la argumentación.

A continuación, se analizan tres ejemplos, uno de cada subtipo de DP, con el objetivo de mostrar la incidencia de estos enunciados en la construcción de sentido: Considérese, en primer término, (6), extraído de la traducción al español de La gaviota, de Chéjov ([1896] 1997:21):

(6) Nina: iQué cuentos maravillosos tiene! [Trigorin]. Tréplev (con frialdad): No sé, no los he leído.

Si se analiza solamente el decir atribuido a Tréplev, se observará que este escenifica una negación polémica (Ducrot, 1984), la cual evoca dos PdV: uno rechazado (" $\lambda$ leer PLT $\lambda$ saber"), otro con el que L (Tréplev) queda homologado (" $\lambda$ neg. leer PLT $\lambda$ neg. saber"). Hasta aquí, el enunciado podría interpretarse simplemente como una constatación de la no lectura de los materiales. Sin embargo, la muestra posee una DPE ("(con frialdad)") que, por su parte, incorpora un tercer PdV que podría formularse en términos de "falta de interés PLT con frialdad". Esta perspectiva enunciativa, crucial para la identificación del sentido de la réplica, induce a reconocer que la no lectura de los cuentos se funda en el desinterés que posee el personaje por la obra de Trigorin. Es más, el PdV que aporta la didascalia conduce a descartar la idea de que la réplica 
representa un acto de constatación, sino que, por el contrario, el enunciado a cargo del personaje debe interpretarse como orientado a dar por terminada la discusión sobre la literatura producida por su rival. Nótese, al respecto, que sin la DPE este sentido no podría actualizarse. Asimismo, es importante decir que, si se considera la enunciación de Tréplev en el universo de ficción, el PdV evocado por la didascalia se produciría a nivel suprasegmental (a partir del modo de decir) y, en ese sentido, funcionaría como una perspectiva mostrada en su expresión que operaría como un marco. En términos formales, el sentido de la réplica considerando su enunciación en el contexto de la situación dramática podría describirse de este modo:

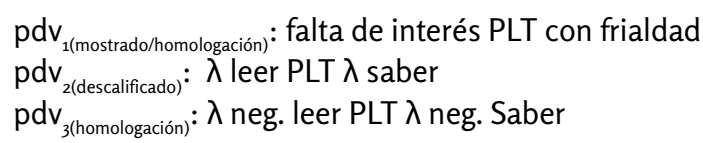

A los efectos de que se comprenda la formalización, debe decirse que cada una de las líneas reproduce uno de los PdV plasmados por la réplica del personaje. Entre paréntesis y con subíndice se señala el posicionamiento del Locutor (aquí, Tréplev) frente a ese PdV. Las diferencias en la sangría buscan reflejar la relación jerárquica entre los PdV (básicamente, se pretende mostrar si alguno de ellos funciona como un marco). Finalmente, la sigla "neg." simboliza "no" o "negación".

Como puede observarse entonces, la DPE aparece para acotar los posibles sentidos del enunciado del personaje. En efecto, incorpora una perspectiva enunciativa, que en el intercambio oral ficcional se convocaría desde la prosodia, que precisa las características que adquiere la réplica (un discurso que se muestra como poco entusiasta). Estas cualidades particulares, por su parte, validan la enunciación a cargo de Tréplev: la no lectura se presenta como motivada por la falta de interés.

En segundo término, se analizará un fragmento del diálogo que se establece entre John Proctor y su mujer Elizabeth en la traducción al español de Las brujas de Salem de Miller, pieza de discurso que posee una DPI:

(7) Proctor: [...] Tendría que haberte hecho callar a gritos la primera vez que me contaste tus sospechas. Pero fui débil, y confesé como un cristiano. ¡Confesé! Por algún sueño que tuve, debí de confundirte con Dios aquel día. Pero no ere Dios, Elizabeth, no señor, iy más valdrá que lo recuerdes! Busca más bien algo bueno en mí, y no me juzgues.

Elizabeth: No te juzgo. El magistrado que te juzga está en tu mismo corazón. Siempre te he considerado un hombre bueno, John. (Con una sonrisa) Aunque un tanto desorientado. ([1953] 1997:68-69)

A los efectos de que se comprenda el análisis, es importante decir que el segmento citado reproduce una conversación privada entre Proctor y Elizabeth, matrimonio que está enfrentando una acusación de brujería realizada por un grupo de jóvenes del pueblo. Al respecto, el participio "desorientado" con el que concluye el ejemplo debe leerse como "poco atento". En efecto, la crítica que realiza Elizabeth, que se analizará a continuación, alude a que su marido no reparó en que Abigail, una de las jóvenes, podía reaccionar con despecho (inventando la acusación) luego de que él rechazara su propuesta de establecer un vínculo sentimental con ella.

Dicho esto, en lo que sigue se examinará la última porción del ejemplo ("Siempre te he considerado un hombre bueno, John. (Con una sonrisa) Aunque un tanto desorientado") buscando determinar la función de la didascalia en la construcción de sentido. En principio, hay que decir que la réplica atribuida a Elizabeth posee dos segmentos que reproducen una estructura concesiva. Estos, por su parte, escenifican dos PdV: 
con el primero, L emerge adoptando una posición de aceptación; con el segundo, una de identificación. En este caso en particular, la primera porción del enunciado plasma un PdV del tipo "Proctor ser bueno PLT merecer aprecio" que insta a leer el fragmento como un elogio. La segunda, sin embargo, introduce un PdV que podría formularse en términos de "Proctor ser desorientado PLT neg. merecer aprecio" que conduce a leer la expresión como una crítica. Como sucede siempre en este tipo de estructuras, la fuerza argumentativa del enunciado recae sobre el segundo segmento, a partir del cual se establecen las continuidades discursivas. En cuanto al valor específico del conector, "aunque" opera como un elemento que mitiga el carácter elogioso del primer segmento.

No obstante, interesa analizar aquí qué significado aporta la DPI y cómo esta ajusta el sentido de crítica del enunciado. Al respecto, "(Con una sonrisa)" puede describirse en función del encadenamiento argumentativo "afecto PLT con una sonrisa", $\mathrm{PdV}$ que, como se viene argumentando, precisa la escenografía de la continuidad discursiva. Así, en (7) la perspectiva enunciativa introducida por la didascalia construye una escena de habla afectuosa, que, por su parte, debilita el valor crítico del enunciado. En otras palabras, la acción de la DPI induce a leer el señalamiento de Elizabeth sobre el carácter de su marido como un juicio que se produce desde el amor que ella siente por él. Por cierto, es importante decir que, si la didascalia no estuviera presente, este valor no quedaría actualizado y la expresión podría ser interpretada como una declaración fuertemente crítica. Finalmente, y si se considera la enunciación del personaje en el universo de ficción, el PdV aportado por la DPI sería evocado a nivel prosódico, esto es, con un tono particular (más allá de la sonrisa) y, en ese sentido, constituiría una perspectiva mostrada que enmarca el segundo segmento del enunciado. Este aspecto del sentido, como se detalló, no puede ser repuesto por la réplica.

Para concluir con el análisis, se presenta a continuación una descripción formal del sentido que el segmento tendría considerando su enunciación en el marco de la situación dramática:

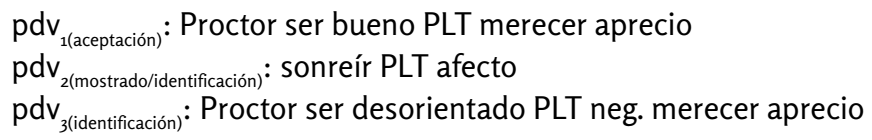

Como mostró la descripción del ejemplo, la DPI no aparece simplemente para indicar que el personaje sonríe mientras enuncia, sino para ajustar el sentido lingüístico de la porción de discurso a su derecha.

En tercer término, se presenta un caso de DPF con el objetivo de detallar su funcionamiento particular:

(8) (a) Enfermera: Pero no hay rosas. Hasta que un día llega un ramo para mí, y lo pongo en un florero creyendo que me lo mando él. Las mismas rosas del barco.

(b) Paciente: Quiere decir que apenas casada y está pensando en otro.

(c) Enfermera: Él llega y me pregunta quién me las mandó.

(d) Paciente: ¿Quién fue?

(e) Enfermera: No se sabe y un día me doy cuenta de que estoy esperando fa-

milia. (Se quita la cofia, cae desordenadamente su pelo gris.)

(f) Paciente: ¿A su edad?

(g) Enfermera: Es verdad, me olvidaba de eso. Es que en este sueño siempre me veo joven, de veinte... o veinticinco años [...]. (Puig, [1987] 2009:139) 
En (8), fragmento extraído de Misterio del ramo de rosas, de Puig, la Enfermera le cuenta a su compañera un sueño que tuvo, en el que, en determinado momento, el personaje se descubre embarazada. Frente al relato de esta situación, en (8f), la Paciente, utilizando la edad de la Enfermera como argumento, cuestiona la factibilidad de este suceso. En lo que sigue, se analizará en detalle el tipo de interrogación que efectúa la Paciente y su relación con la DPF presente en la réplica previa de la Enfermera.

En principio, el enunciado didascálico evoca un PdV que puede formularse en términos de "mostrar pelo gris PLT exhibir edad avanzada". Este, tal como se intentará explicar, tiene una incidencia fundamental en la réplica siguiente, ya que permite leer en (8f) la existencia de un punto de vista mirativo vehiculizado por la estructura interrogativa. Al respecto, es importante aclarar que, tradicionalmente, se denomina miratividad a la actitud de sorpresa que manifiesta un hablante frente a información nueva o inesperada. No obstante, para el EDAP, que, como se recordará, constituye una perspectiva no referencialista ni intencionalista de la significación, esta definición es problemática, ya que hace intervenir la categoría de sujeto empírico. En su lugar, para este enfoque teórico, este fenómeno se concibe como la evocación de una representación argumentativa de la causa de la enunciación en la que $\lambda$ aparece descubriendo algún evento o desmantelando una creencia previa. La manera en la que queda mostrado $\lambda$ frente al nuevo conocimiento adquirido es aquello que explica las características que asume la enunciación (García Negroni y Libenson, 2018).

Así las cosas, en (8f), el enunciado de la Paciente, gracias a la acción de la didascalia, insta a recuperar un marco de discurso previo $(\operatorname{ver}(\lambda)$ X PLT descubrir $(\lambda)$ Y) que emerge como la causa fundante de la enunciación. En otras palabras, la pregunta del personaje se presenta motivada por el hecho de que ella es mostrada constatando la edad de la Enfermera y, por lo tanto, descubriendo con sorpresa su imposibilidad de quedar embarazada. En ese sentido, la presencia del PdV mirativo es fundamental en la construcción del sentido del enunciado, ya que su identificación permite leer que (8f) no es simplemente una pregunta, sino una enunciación fuertemente desconfiada que surge porque la Paciente observa que la edad de su compañera vuelve su relato poco verosímil. En términos del EDAP, el sentido de la réplica en (8f) admite la siguiente descripción:

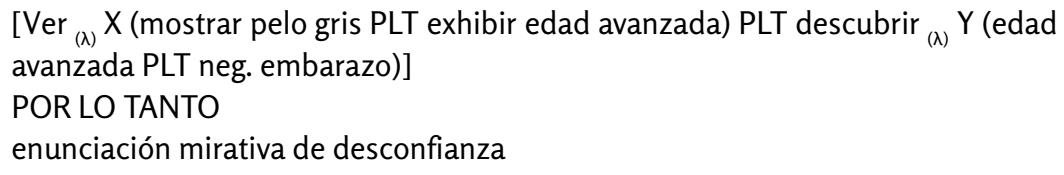

Con el objetivo de que se comprenda la formulación, es importante decir que entre corchetes se grafica el encadenamiento argumentativo que funciona como marco de discurso. En ese sentido, el conector dialógico "POR LO TANTO" (García Negroni, 2018a) busca mostrar, justamente, que las características que adquiere la enunciación emergen en términos de una respuesta dialógica frente a ese marco.

Como puede observarse entonces, la didascalia cumple la función de ajustar el sentido de la réplica que le sigue precisando la escenografía del enunciado. Específicamente, la DPF colabora en la construcción de una representación argumentativa de aquello que causó la enunciación y esto induce a reconocer que el enunciado evoca una escena de habla en la que, frente a la constatación directa de la edad de la Enfermera, la Paciente no puede hacer otra cosa que cuestionar la factibilidad del embarazo. De esta forma, la didascalia incorpora una instrucción dialógico-causal sobre la que se funda y se legitima la enunciación: 
La enunciación es de sorpresa y desconfianza PORQUE veo $(\lambda)$ que la Enfermera tiene una edad avanzada.

Al respecto, es importante aclarar que, si bien (8f) posee una estructura interrogativa que funciona como la marca del PdV mirativo, la mera presencia de los signos de pregunta no le da a la enunciación el carácter de fuerte desconfianza que aquí adquiere. Es la DPF en (8e), como se mostró, la que activa esta lectura, específicamente, precisando el valor particular que aquí tiene la curva de entonación interrogativa. Nótese, además, que aquí también la didascalia aparece para incorporar un aspecto de sentido propio de la comunicación oral que la réplica no puede construir por sí sola.

Finalmente, y a los efectos de proponer una descripción completa del funcionamiento enunciativo del ejemplo, la DPF también establece un lazo dialógico con el fragmento de discurso que la antecede, en este caso, modificando la dirección de la argumentación. En efecto, si “No se sabe y un día me doy cuenta de que estoy esperando familia" orienta el discurso a encadenamientos en los que se continúe con el relato del embarazo, la perspectiva evocada por la didascalia anula la aparición de estas posibles continuidades, ya que la exhibición de la edad del personaje muestra la imposibilidad de su relato.

En síntesis, los ejemplos presentados muestran el rol fundamental que las DP tienen en el proceso de construcción de sentido. Como quedó dicho, estos enunciados se especializan en plasmar un PdV que, además de describir algún aspecto de la situación dramática, ajusta el sentido de las réplicas (en particular, define su escenografía). Para ello, instan a leer en el discurso que modifican una perspectiva enunciativa que en el intercambio oral ficcional se evocaría a partir de elementos suprasegmentales, los cuales las réplicas no pueden reponer por sí mismas. Esta hipótesis, por su parte, permite explicar la frecuencia de aparición de estos discursos, aspecto hasta ahora no estudiado por la bibliografía especializada. En efecto, si la caracterización propuesta aquí es correcta, las DP surgirían solo en aquellos casos en los que el enunciado atribuido al personaje no pueda evocar su escenografía por sus propios medios (o cuando esta no se especifique en el enunciado que le sigue como respuesta). Esto revelaría, entonces, por qué no toda réplica viene acompañada de una DP.

Así las cosas, es importante decir que la hipótesis aquí presentada difiere significativamente de la explicación que los estudios tradicionales proveen sobre el funcionamiento de estos discursos. Como se detalló más arriba, las didascalias tienden a ser consideradas como enunciados que informan sobre el contexto en el que se produce el dialogo teatral. Sin embargo, se observó que estas, al menos las DP, no señalan "las condiciones concretas del uso de la palabra" (Ubersfeld, 1989:17), sino que su función es la de ajustar y precisar el propio significado lingüistico de las réplicas.

\section{Conclusión}

Como se observó a lo largo de este trabajo, las didascalias son uno de los fenómenos más estudiados en el campo de la teatrología. Sin embargo, las dos tradiciones que se ocuparon de caracterizar este tipo de enunciados no lograron arribar a una definición que dé cuenta cabalmente de su especificidad ni de sus funciones en el proceso de construcción de sentido de todo TD. Es más, incluso aquellos autores que se propusieron clasificar estos discursos como una manera de identificar sus diversos roles y atributos tampoco arribaron a una taxonomía satisfactoria. En efecto, ya sea porque no proveen una caracterización lingüística rigurosa o porque no utilizan un criterio uniforme de clasificación, estas tipologías terminan siendo poco operativas al momento de ubicar un enunciado didascálico dentro de una categoría específica. 
Así las cosas, este artículo buscó arrojar luz sobre el problema mediante la identificación de la naturaleza particular de los enunciados didascálicos. Para ello, y a partir de la adopción del Enfoque dialógico de la argumentación y la polifonía enunciativa como marco teórico, se indicó, en primer término, que es necesario reconocer que existen una serie de diferencias formales y funcionales en el discurso didascálico, diferencias que vuelven imprescindible la construcción de una taxonomía que permita aislar nítidamente las propiedades de cada uno de los subtipos. En función de ello, en este trabajo se identificaron cinco clases de didascalias: las paratextuales (exteriores a la narración y ocupadas en construir un marco de lectura), las estructurales (encargadas de dividir el texto en secuencias), las generales (oraciones autónomas a partir de las cuales se construye el contexto de enunciación en el que se desarrolla el diálogo teatral), las atributivas (que señalan la identidad del Locutor a cargo de la réplica) y las particulares (didascalias sin autonomía sintáctica que se especializan en colaborar en la construcción de la escenografía del discurso atribuido a los personajes). Además, dentro de esta última categoría, las distintas posiciones en las que se encuentran estos enunciados condujeron a distinguir entre las didascalias particulares externas, las internas y las finales.

Dejando de lado los rasgos específicos de cada categoría, interesa destacar aquí que la taxonomía propuesta toma en consideración las características lingüísticas distintivas de cada subtipo y, además, utiliza un criterio de clasificación uniforme. Como quedó dicho, se considera que estas son ventajas considerables en relación con otras de las tipologías que se proponen explicar el funcionamiento de estos enunciados.

Finalmente, se sugirió que en ningún caso las didascalias deberían concebirse como acotaciones escénicas, como enunciados que indican la forma en la que un TD debería ser llevado a escena. Al respecto, se mostró que toda didascalia posee un valor metadiscursivo y que es la propia enunciación dramática la que constituye el objeto de ese comentario. Dicho de otro modo, las didascalias aparecen para guiar la lectura: ordenan la acción en secuencias, construyen y modalizan la situación dramática y ajustan el sentido de las réplicas. En cuanto a este último punto, se probó además que, al menos las didascalias particulares, no están simplemente al servicio de aportar información extralingüística (como afirman los autores que se dedicaron a estudiar estos enunciados). Por el contrario, estas expresiones cumplen un rol esencial en la determinación del significado lingüístico de las réplicas, ya que colaboran en la definición de la escenografía, de la escena de habla generada por el enunciado orientada a validar la propia enunciación.

Quedará pendiente para futuros trabajos cotejar la taxonomía aquí presentada con enunciados didascálicos presentes en otros TD a los efectos de poder evaluar tanto su productividad como la caracterización propuesta para cada subtipo. 


\section{Dibliografía}

\section{Fuentes}

» Beckett, S. ([1957] 2006). Final de partida. Buenos Aires: Tusquets Editores.

»Brecht, B. ([1943] 1967). El alma buena de Se-Chuan. Buenos Aires: Ediciones Nueva Visión.

"Chéjov, Á. (1997). La gaviota. Las tres hermanas. El tío Vania. Buenos Aires: Losada.

»Cossa, R. (2000). El avión negro. La nona. No hay que llorar. Buenos Aires: Ediciones De la Flor.

»Ibsen, H. (2008). Un enemigo del pueblo. Casa de muñecas. Buenos Aires: Punto de encuentro.

" Kane, S. (2009). Ansia. 4.48 psicosis. Buenos Aires: Losada.

» Kartun, M. (2006). Chau Misterix. El partener. La Madonnita. La suerte de la fea. Buenos Aires: Losada.

» Miller, A. ([1953] 1997). Las brujas de Salem. Barcelona: Tusquets Editores.

» Minyana, P. ([1986] 1998). “Inventarios”. En Teatro francés contemporáneo II (pp. 129-162). Montevideo: Ediciones Trilce.

» Pinter, H. ([2005). El cuidador. Los enanos. La colección. Buenos Aires: Losada

»Puig, M. (2009). Teatro reunido. Buenos Aires: Entropía.

"Strindberg, A. ([1889] 2011). La señorita Julia. El padre. Buenos Aires: Losada.

》Weiss, P. ([1964] 2010). Marat-Sade. Buenos Aires: Adriana Hidalgo Editora.

\section{Bibliografía específica}

" Abirached, R. (1994). La crisis del personaje en el teatro moderno. Madrid: Asociación de Directores de Escena de España.

»Andjelkovic, S. (2008). “Le texte didascalique: dialogue de l'auteur avec sa propre fiction dans les drames de Biljana Srbljanović et Ivana Sajko". Revue des études slaves 79(1-2), 119-131.

» Aikhenvald, A. (2004). Evidentiality. Oxford, Oxford University Press.

"Anscombre, J.-C. y Ducrot, O. (1994). La argumentación en la lengua. Madrid: Gredos.

» Bajtín, M. ([1979] 2002). Estética de la creación verbal. México: Siglo XXI.

» Bobes Naves, C. (2004). “Teatro y semiología”. Arbor CLXXVII 699-700, 497-508.

"Caldiz, A. (2015). "Subjetividad, prosodia y ponencias académicas. A propósito del ethos autoral surgido de la lectura en voz alta". En García Negroni, M.M. (ed.), Sujeto(s), alteridad y polifonia. Acerca de la subjetividad en el lenguaje y el discurso (pp. 51-76). Buenos Aires: Ampersand/ Editorial de la Facultad de Filosofía y Letras de la Universidad de Buenos Aires. 
"Caldiz, A. (2019). "Puntos de vista evidenciales y entonación". Antares 11(23), 53-74.

»Carel, M. y Ducrot, O. (2005). La semántica argumentativa: una introducción a la teoría de los bloques semánticos. Buenos Aires: Colihue.

»Carrasco Muñoz, I. (1980). “Naturaleza y función de las acotaciones (a propósito de Buero Vallejo)". Estudios Filológicos 15, 39-50.

"Conde, L. (2017). “Didascalias: la piel del teatro". Estudios de Teoría Literaria. Revista digital: artes, letras y humanidades 11, 15-27.

"Conde, L. (2018). “La máquina teatral y sus fantasmas". El jardín de los poetas. Revista de teoría y crítica de poesía latinoamericana 6, 35-52.

"De Marinis, M. (2005). En busca del actor y del espectador. Buenos Aires: Galerna.

》De Toro, F. (2008). Semiótica del teatro. Buenos Aires: Galerna.

"Dompeyre, S. (1992). "Étude des fonctions et du fonctionnement des didascalias”. Pratiques: linguistique, littérature, didactique 74, 77-104.

»Ducrot, O. (1984). El decir y lo dicho. Buenos Aires: Paidós.

»Eco, U. (1981). Lector in fabula. Barcelona: Lumen.

» Fitzpatrick, T. (1987). "Análisis de textos dramáticos y de espectáculos: hacia un modelo teórico". Semiosis 19, 191-211.

» Fobbio, L. (2009). El monólogo dramático: interpelación e interacción. Córdoba: Comunicarte.

» García Barrientos, J. L. (2009). “Acotación y didascalia: un deslinde para la dramaturgia actual en español”. En Barrientos, J et al. (coord.), En buena compañía. Estudios en honor a Luciano García Lorenzo (pp. 1125-1139) Madrid: Consejo Superior de Investigaciones científicas.

" García Barrientos, J. L. (2012). Cómo se comenta una obra de teatro. México: Paso de gato.

» García Negroni, M. M. (2016a). “Polifonía, evidencialidad y descalificación del discurso ajeno. Acerca del significado evidencial de la negación metadiscursiva y de los marcadores de descalificación". Letras de Hoje 51(1), 7-16.

»García Negroni, M. M. (2016b). “Discurso político, contradestinación indirecta y puntos de vista evidenciales. La multidestinación en el discurso político revisitada". Revista ALED 16(1), 37-59.

"García Negroni, M. M. (2018a). “Argumentación y puntos de vista evidenciales citativos. Acerca de la negación metadiscursiva en el discurso político". Oralia 21, 223-236.

»García Negroni, M. M. (2018b). “Argumentación y puntos de vista evidenciales: acerca del condicional citativo en el discurso periodístico y en el discurso científico". Boletín de lingüística XXX (49-50), 86-109.

"García Negroni, M. M. (2019). "El enfoque dialógico de la argumentación y la polifonía, puntos de vista evidenciales y puntos de vista alusivos". Rilce. Revista de Filología Hispánica 35(2), 521-549.

"García Negroni, M. M. y Libenson, M. (2018). “iAl final tenías plata! Acerca de las causas mirativo-evidenciales de la enunciación". Trabajo presentado en el Coloquio Internacional sobre Evidencialidad, Universidad Autónoma de México. 
» García Negroni, M. M. y Libenson, M. (2019). “A propósito de las causas dialógicas de la enunciación. El caso de las enunciaciones mirativas con el marcador Mirá". Trabajo presentado en el $6^{\circ}$ Congress of Discourse Markers in Romance Languages, Università di Bergamo.

» Genette, G. (1989). Palimpsestos. La literatura en segundo grado. Madrid: Taurus.

» Helbo, A. (2012). El teatro: ¿texto o espectáculo vivo? Buenos Aires: Galerna

"Hermenegildo, A. (1991). “Los signos condicionantes de la representación: el bloque didascálico". En González del Valle, L. T. y Baena, J. (eds.), Critical Essays on the literatures of Spain and Spanish America (pp. 121-131). Colorado: Society of Spanish and Spanish-American Studies.

"Ingarden, R. (1971). "Les fonctions du langage au theatre" (Trad. C Bobes Naves). Poétique $8,531-538$.

》Issacharoff, M. (1981). “Texte théâtral et didascalecture”. MLN 96(4), 809-823

"Jakobson, R. (1975). Ensayos de lingüística general. Barcelona: Seix Barral.

"Lasso Osorio, J. (2013). Textos dramáticos y traducción teatral. Trabajo de grado. Programa de Artes Dramático, Departamento de Artes Escénicas, Facultad de Artes integradas, Universidad del Valle, Santiago de Cali.

» Llagostera, L. (2015). "El para - texto: un auxiliar para el análisis del texto dramático y espectacular en la era del teatro post dramático". Trayectoria. Práctica Docente en Educación Artística 3, s/p.

»López, L. (2013). “Reescrituras teatrales contemporáneas de los clásicos: efectos y perspectivas críticas”. En López, L. (coord..), Topología de la crítica teatral III. Reescrituras críticas de la escena contemporánea (pp. 51-62). Buenos Aires: IUNA.

" Maingueneau, D. (1999). “Ethos, escenografía, incorporación”. En Amossy, R. (dir.), Images de soi dans le discours (pp. 75-100). Lausanne: Delacahux el Niestlé.

» Maingueneau, D. (2002). Problemas de ethos. Pratiques, 113/114, 55-67.

"Pavis, P. (1988). Diccionario de teatro: dramaturgia, estética, semiología. Barcelona: Ediciones Paidós.

"Petitjean, A. (2011). "Pour une problématisation linguistique de la notion de genre: I'exemple du texto dramatique". Écho des études romanes VII(2),5-20.

»Prochazka, M. (1997). "Naturaleza del texto dramático". En Bobes Naves, C. (comp.), Teoría del teatro (pp. 57-81). Madrid: Arco.

»Rubio Montaner, P. (1990). “La Acotación escénica. Transferibilidad y Feedback. Caracteres funcionales, sustanciales y pragmáticos". Anales de filología hispánica 5, 191-201.

» Ryngaert, J. P. (2004). Introducción al Análisis teatral. Buenos Aires: Ediciones Artes del Sur.

»Sanchis Sinisterra, J. (2002). La escena sin límites. Fragmentos de un discurso teatral. Guadalajara: Ñaque.

»Schmidhuber de la Mora, G. (2009). “Dramaturgia en las didascalias”. Revista Teatro/Celcit 35-36, 192-209.

»Thomasseau, J. M. (1997). "Para un análisis del para-texto teatral Nota 1: Genealogía del concepto de didascalia”. En Bobes Naves, C. (comp.), Teoría del teatro (p. 83-118). Madrid: Arc 
» Ubersfeld, A. (1989). Semiótica teatral. Madrid: Cátedra.

»Vaisman, L. (1979). “La obra dramática: un concepto operacional para su análisis e interpretación en el texto”. Revista Chilena de Literatura 14, 5-22.

"Veltrusky, J. (1997). "El texto dramático como uno de los componentes del teatro”. En Bobes Naves, C. (comp.), Teoría del teatro (pp. 31-55). Madrid: Arco.

»Villegas, J. (1991). Nueva interpretación y análisis del texto dramático. Ottawa: Girol Books.

»Zich, O. (1931). Estetika dramatického umení. Pracha: Melantrich

»Zucchi, M. (2019). "Una clasificación del discurso didascálico desde una perspectiva polifónica de la enunciación”. Revista Letrónica 11(4), 452-464. 